\title{
Horizontal vs. Vertical: How the Orientation of a Large Interactive Surface Impacts Collaboration in Multi-Surface Environments
}

\author{
Lili Tong $^{1}$, Aurélien Tabard ${ }^{2}$, Sébastien George ${ }^{3}$, Audrey Serna $^{1}$ \\ ${ }^{1}$ Univ Lyon, CNRS, INSA-Lyon, LIRIS, UMR5205, F-69621, Villeurbanne, France \\ ${ }^{2}$ Univ Lyon, CNRS, Université Lyon 1, LIRIS, UMR5205, F-69622, Villeurbanne, France \\ ${ }^{3}$ UBL, Université du Maine, EA 4024, LIUM, 72085 Le Mans, France \\ \{lili.tong, aurelien.tabard, audrey.serna\}@insa-lyon.fr, sebastien.george@univ-lemans.fr
}

\begin{abstract}
Defining the form factor and set-up of surfaces, i.e., their size, position, and orientation, is one of the first decisions made when designing multisurface environments (MSE). To support these choices, we conducted a study on how the orientation of a large display used alongside tablets impacts collaboration. Previous research involving only one interactive surface shows that display orientation changes how people interact with the display, the way they position themselves, or look at each other. Our study shows that in a MSE setting, the orientation of a large surface has a different impact: (1) it nuances previous results showing that horizontal surfaces are better for collaboration. (2) it impacts the way activities are conducted. The horizontal condition leads to more implicit coordination and balanced interaction with the large display, but to less structured work, while in the vertical condition, group coordination is more explicit and is structured around one main interactor. Compared to previous work, we also propose a more structured, comprehensive and detailed analysis grid for collaboration in MSE. Finally, based on our results, we derive recommendations for MSE design.
\end{abstract}

Keywords: Collaboration; coordination; Multi-Surface Environments (MSE): tabletops: tablets; display orientation.

\section{Introduction}

Multi-surface environments (MSE), i.e., the combination of devices into a seamless information space, have shown benefits for co-located collaboration [1]. Collaborative MSE are often composed of large displays acting as a shared space to coordinate efforts, and handheld devices used as personal spaces for individual tasks. The introduction of personal devices alongside large shared surfaces tends to improve efficiency of individual tasks within larger collaborative activities [32]. This has proved to be especially useful in educational contexts [28], emergency response planning [5], and gaming $[9,26]$. 
The form factor, i.e. size, orientation, overall shape, and configuration of surfaces in a MSE is generally decided early in projects. Yet these factors can have profound effects on how people interact in these environments. For instance, Zagermann et al. [35] recently showed that as the size of tabletops increases in a MSE, collaboration quality or sensemaking results decrease, since the larger screen diverts users' attention away from their collaborators and towards the shared display. Closer to our concerns, Rogers and Lindley [23] showed that horizontal displays supported a greater awareness of participants' activities compared to vertical ones.

In this paper, we investigate how the orientation of a large shared interactive display impacts collaboration in a Multi-Surface Environment. We revisit the question originally framed by Rogers and Lindley with a single surface, within a richer ecosystem of devices with more sophisticated inputs, i.e., multi-touch instead of a mimio pen. To understand the impact of display orientation, both on interaction and coordination aspects, we conducted a study in which twelve groups of three participants carried out a collaborative problem solving activity with tablets, associated with a 55" shared display, both in vertical and horizontal position (Fig. 1).

Our results show that multi-surface environments reduce the differences between the horizontal and vertical conditions compared to previous research studying group work with horizontal and shared surfaces [15, 22, 23]. Participants maintained a good level of awareness, created a similar amount of content and discussed in the same proportions in both conditions. However, we observed differences in activity organization between the two conditions. Participants in the horizontal condition acted more equally using both explicit and implicit coordination mechanisms, leading to anticipation, assistance and parallel work. In contrast, the vertical condition led to the emergence of a more structured activity with a main interactor and explicit distribution of labor among the group. We describe in detail the awareness mechanisms at play and their consequences on activity organization. Overall, our results weigh in, confirm, contradict, and extend previous work.

\section{Related Work}

We focus on collaboration in multi-surface environments. We are especially interested in understanding the interplay between the form-factors of devices, their affordances, and people's behaviors at both the individual and group level.

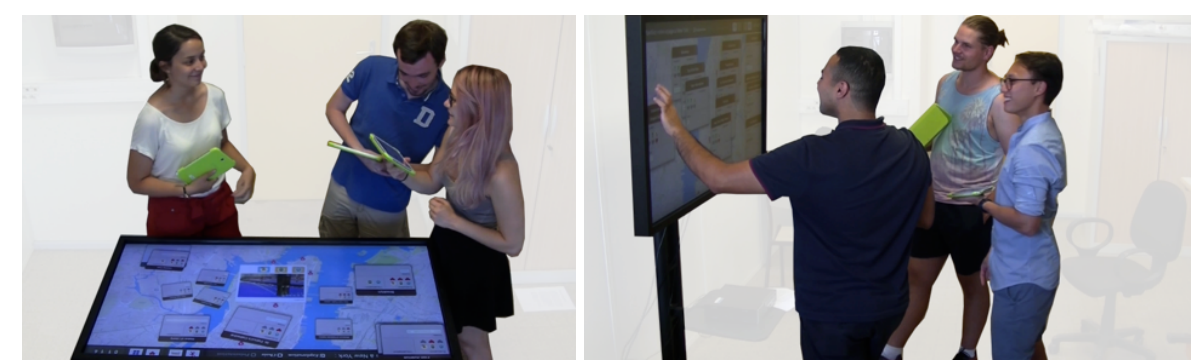

Fig. 1. A large interactive surface used alongside tablets in a problem solving activity. Left: the horizontal condition. Right: the vertical condition. 


\subsection{Multi-surface environments}

In a number of ways, Weiser's vision of ubiquitous computing environments in which people can seamlessly interact across devices [33], has made its way into our homes, workplaces, and learning environments [4]. These Multi-Surface Environments are particularly suited for conducting complex collaborative problem solving activities involving rich data exploration. Examples range from urban planning [28], to basin (oil/gas) exploration [25], as well as emergency response planning [5]. Games are another example of collaborative activities that can benefit from MSE $[9,26]$.

When looking at devices independently, commonly held views are that: (1) large shared surfaces are well suited to co-located collaborative activities [27]; (2) tabletops enable more equitable participation [20]; and (3) handheld devices support mobility with pervasive access to information, support planning and enable monitoring of activities [21]. However, the question of how to support effective collaboration in MSE is still open [5].

\subsection{The impact of device form factors on collaboration}

When building MSE, decisions concerning the size, form and orientation of devices have to be made early on in the design process. These factors are often considered implicitly, or intuitively since data is scarce in the domain. The complexity of changing form factors may explain why few papers discuss their impact on interaction, even though these factors profoundly shape the affordances of devices. To mitigate this problem Inkpen et al. used a paper-based prototype to display orientation, size, and user arrangements [15]. They found that although participants felt the horizontal display was more natural and comfortable for collaboration, working with a vertical display tended to be more time-efficient.

Rogers and Lindley were among the first to study impact of device orientation on interaction and collaboration [23]. Through two experiments, they showed that horizontal displays are better at supporting collaboration, as they promote more suggestions and idea generation, while also leading to more role switches and greater awareness of others' actions. The authors proposed two reasons (1) the input device, a mimio pen, was easier to pass among users over the horizontal table, and (2) it was harder to input data on the vertical display while standing. More recent work by Potvin el al. [22] comparing vertical and horizontal multi-touch displays found that the horizontal surface encouraged more equal physical interactions among participants with the shared display, as well as equal verbal participation, which differs from Rogers and Lindley's study. Al-Megren et al. also showed that the vertical configuration was more likely to cause muscle fatigue comparing to the horizontal configuration [2].

All these previous studies focused on a single screen. Since Rogers and Lindley's study in 2003, large multi-touch displays, smartphones and tablets have become pervasive, raising the question of how people behave and collaborate in such MultiSurface Environments. Compared to previous work, devices like smartphones or tablets make input much more efficient, which should change collaboration by distributing control more evenly. The same applies to input on large devices which is now fast, reliable and multi-touch, meaning that anybody can take control of a shared screen without any limitation. 


\subsection{Coordination mechanisms}

To effectively design collaborative activities in MSE, it is important to acquire a deep understanding of complex interactions occurring between users and devices, and individuals within a group [29]. In this context, coordination of individual actions within groups is crucial when pursuing collaborative activities. Malone and Crowston define coordination as "the act of managing interdependencies between activities performed to achieve a goal" [18]. In coordinated work, participants strive towards a shared goal dealing with time and organizational constraints. Several mechanisms are used in the coordination process such as awareness, regulation, information sharing and discussion.

Awareness of individual and group actions is crucial for a successful collaborative work. For Schmidt [24], awareness refers to actors "taking heed of the context of their joint effort". It also refers to monitoring practices of others and acting in a way that makes aspects of activity visible. Yuill and Rogers do not limit awareness to actions but extend it to situations where people "have ongoing awareness of the actions, intentions, emotions and other mental states of other interactants" [34]. In our work, we refer to awareness as a state of mutual consciousness allowing structuration of an activity, avoiding duplication of work and facilitating group progress and activity coordination. As Hornecker et al. state, with a good level of awareness, little verbal communication is used in coordinating activity, and assistance and anticipation actions arise [14]. On the contrary, a lack of awareness can negatively impact coordination. Interferences, "unintended negative influence on another user's actions" according to Hornecker et al. [14], can arise with multi-user devices when two or more participants try to perform incompatible actions (e.g. attempt to drag the same object, or select two inconsistent features).

Regulation builds upon awareness and relates to people's ability to plan, monitor, evaluate and regulate the joint activity [29, 31]. The concept of regulation is extensively used in the learning and psychology literature for analyzing collaborative behaviors [10]. In HCI, regulation is observed in terms of activity organization. Studies analyze the way group members elaborate strategies [35], adopt roles and distribute or share labor [23] to understand this meta-level of coordination and how it relates to the overall activity.

Finally, information sharing and discussions are also required in coordination processes. Information sharing involves building a common ground [7], which means that members collaborate in ensuring understanding and in grounding their mutual knowledge and assumptions. It also contains sharing information on physical objects such as documents and materials. The number and type of discussion occurring during group work are often considered for analysis of collaboration [11].

\subsection{Group formations and mobility in collaboration}

Physical formations also influence group behavior, which in turn shapes the physical formations. The notion of Facing formation, or F-formation, was introduced by Kendon [17] to describe how people adjust their position and orientation to interact together and jointly manage their attention. Although our study participants were not 
very mobile, we nonetheless paid attention to F-formations, proxemics [13], and the social interactions occurring in these arrangements. Here, the form factor and physical properties of artifacts also play a role in collaboration [16]. In their study on the use of paper documents, Paul and Luff emphasized particularly how form-factors would afford various levels of micro-mobility, e.g. tilting or flipping devices, which would then shape collaboration [16].

\section{Study}

We have shown that collaboration is important but very fragile and can be influenced by tiny details such as devices configurations, as shown during our case studies. We want to focus orientation to show that this little decision is already very impactful. Previous work [23] shows that horizontal displays offer better opportunities for equal interactions and distributed work coordination among participants. Our question is whether these observations still hold in MSE? In order to study this question, we designed a multi-surface application supporting problem-solving activities. A large surface displays the map, while tablets support information browsing, note taking and bookmarking favorite locations. For our experiment, we implemented a trip planning activity in the application.

\subsection{Pre-study}

In a preliminary study, we explored the impact of display orientation in MSE on individual and collaborative work. Our hypothesis was that the introduction of tablets would decrease the differences between horizontal and vertical conditions by enabling participants to carry out individual activities alongside. The study consisted of a collaborative problem-solving activity made up of an individual phase in which participants analyzed data on their tablets, followed by a collaborative phase in which participants discussed how to come to a collective decision.

The study lasted around 55 minutes. This included 5 minutes of task description and familiarization, 20 minutes for the task in one condition followed by a similar task in the other condition, and 10 minutes of debriefing at the end. We counterbalanced the orientation and the data presented to participants for the two conditions. Six groups of three people participated in the experiment. Within each group, participants knew each other.

\section{Lessons from pre-study}

We found that the horizontal condition seemed to better support coordination among participants. Roles and tasks were most frequently distributed in this condition. Regarding individual tasks, participants inputted equal numbers of notes and arguments.

However, the structured nature of the activity constrained what participants could do. In practice, everyone had to analyze the same data in order to move to the next phase. Moreover, we noticed variations between the two sessions in terms of time spent to complete the task and also in the level of the discussions. Participants spent 
more time in the first session (mean $=19 \mathrm{~m} 24 \mathrm{~s}, \mathrm{SD}=3 \mathrm{~m} 54 \mathrm{~s}$ ) than in the second session $($ mean $=11 \mathrm{~m} 36 \mathrm{~s}, \mathrm{SD}=4 \mathrm{~m} 24 \mathrm{~s})$ with a statistically significant difference $(\mathrm{t}(5)=$ $7.19, \mathrm{p}=0.0008)$. Besides time duration, we also observed that participants discussed task strategy only in the first session and continued to use the same strategy in the second session. Both phenomena made it difficult to compare group behaviors and draw reliable conclusions.

\subsection{Main study}

Based on the observations from the pre-study, we designed a task with fewer constraints and chose a between group experimental design. Our study configuration consisted of three participants, each with a tablet and sharing the multi-touch display. The collaborative activity, which consisted in planning a trip to New York, involved gathering information, analyzing it, and making group decisions.

\subsection{Hypotheses}

Based on the related work and our pre-study, we derived a set of hypotheses ranging from low-level interaction to high-level group organization. At a low-level, we focused on how people interact with devices in MSE while conducting collaborative activities, especially when creating and interacting with content. We hypothesized that:

- H1: the horizontal condition would lead to more balanced physical interactions with the large display within groups;

- H2: the difference in input levels (e.g., notes taken) between the two conditions would not be as pronounced as in prior work that did not include personal devices.

Our second set of hypotheses focused on higher-level activities related to group coordination:

- H3: the horizontal condition would support a higher level of awareness;

- H4: the horizontal condition would support more efficient activity organization;

- H5: the horizontal condition would encourage more communication and discussions.

\subsection{Task design}

The task consisted in planning a trip itinerary to New York with a limited budget, comparable to that used by Rogers and Lindley [23]. Such an activity is open-ended enough to enable various types of group organization. While the task focused on trip planning, the same collaborative mechanisms are at play in any decision-making activities, which can be found in educational contexts (classrooms, museums), professional meetings or show rooms. We chose New York as the degree of knowledge of its landmarks was relatively similar in our target population. Based on their budget, participants had to agree on: how many days they would stay in the city; which hotel they would stay at; which activities they would do; and their itinerary for each day. Once finished, participants had to present the day-to-day outline of their trip. 
The shared screen displayed a map with markers for 15 tourist attractions and 8 hotels (Fig. 2). Participants could push detailed information on their tablet by tapping their avatar on a marker (1). Information provided for each location included: description, price, rating, and feedback from other tourists (5). Using their tablets, participants could individually add locations to their favorites (ㄷ) and take notes (7)). A card per location showed its favorites and notes on the shared screen (2). Four filter buttons on the shared screen were used to show/hide attractions, hotels, favorite locations, location cards (3). A timer in the top right corner reminded participants how much time was left (4).

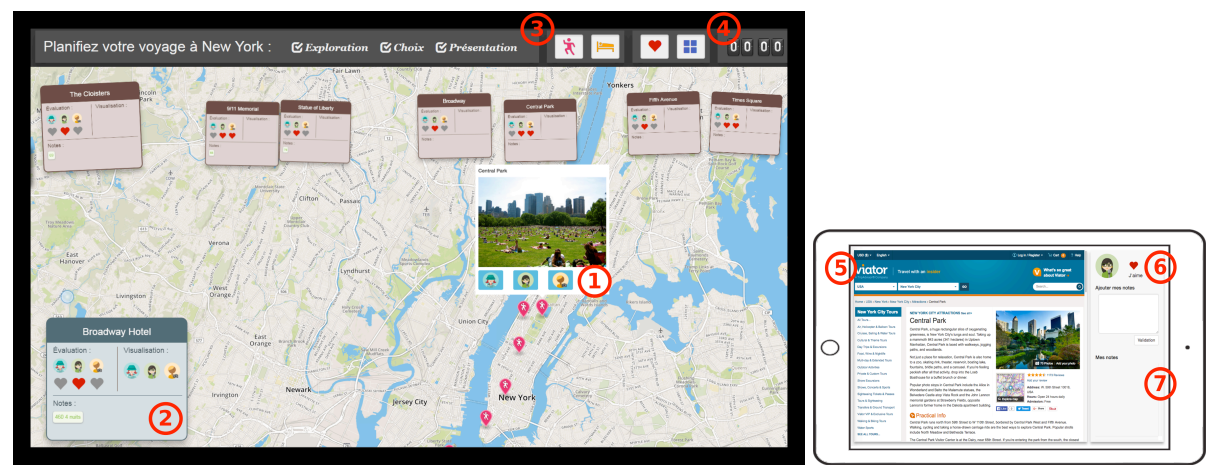

Fig. 2. The application overview. Left: shared surface showing the map + favorite locations. Right: tablets content with details on an attraction.

\subsection{Participants}

We recruited 12 groups of three participants at our university. Group members knew each other. It amounted to 36 participants ( 24 males and 12 females). Participants were between 21 and 31 years old (mean $=26.1 ; \mathrm{SD}=3.28)$. They had different backgrounds including electronic engineering, biology and computer science. All used smartphones, but had never worked or participated studies in MSE.

\subsection{Apparatus}

We used a 55-inch multi-touch display with a resolution of $1920 \times 1080$ pixels in both conditions. The tablets were Samsung Galaxy Note 8 with protective covers. The devices were wirelessly connected to the network. The MSE application was built with Web technologies, and devices communicated via websockets.

\subsection{Procedure}

Based on the literature and our pre-study, we chose a between-group design with the shared display orientation as an independent variable with two horizontal or vertical conditions. In both conditions, participants were standing (Fig. 1). In all we observed six groups of three participants in each condition, which is comparable to similar studies of collaborative work with tabletops $[14,35]$.

The experiment started with 5 minutes of task description and familiarization, and ended with 10 minutes of debriefing. The group had approximately 25 minutes to 
achieve the task, with a timer indicating the time left. However, the task instructions emphasized that the timer was an indication and that participants could spend more or less time depending on how the activity progressed.

\subsection{Data collection}

We collected behavioral data through videos and logs. We used two cameras to record the sessions: one was placed near the ceiling to capture the activity on the shared surface, while the other was placed beside the shared surface to capture the group. We logged interactions with the shared display and the tablets, such as touch events on UI elements, dragging/zooming the map, choosing a location, submitting a note, etc.

\section{$4 \quad$ Analysis method}

To analyze participants' behaviors, we defined a set of indicators detailed in Table 1 The indicators range from low-level actions, e.g. touch events, to higher-level coordination behaviors and group strategies.

\begin{tabular}{|c|c|c|c|}
\hline Behaviors & Data source & & Indicators \\
\hline \multirow{2}{*}{$\begin{array}{c}\text { Interaction with } \\
\text { device }(\mathrm{H} 1 \& \mathrm{H} 2)\end{array}$} & $\log$ & \multicolumn{2}{|c|}{ Notes / Favorite locations / Total number of touch events } \\
\hline & Video & \multicolumn{2}{|r|}{ Touches on shared display per participant } \\
\hline \multirow{3}{*}{ Awareness (H3) } & \multirow{3}{*}{ Video } & Positive & Reaction without request / Complementary action \\
\hline & & Negative & Interference / Verbal monitoring \\
\hline & & Neutral & Verbal shadowing \\
\hline \multirow{2}{*}{$\begin{array}{c}\text { Activity } \\
\text { organization (H4) }\end{array}$} & $\log$ & \multicolumn{2}{|r|}{$\begin{array}{l}\text { Locations checked together at the same time / } \\
\text { Locations checked per person }\end{array}$} \\
\hline & Video & \multicolumn{2}{|r|}{ Discussion on strategy / Duration of the whole task } \\
\hline Discussion (H5) & Video & \multicolumn{2}{|c|}{$\begin{array}{c}\text { Discussion on hotels, attractions, budget and itinerary / } \\
\text { Sharing tablets for discussion }\end{array}$} \\
\hline
\end{tabular}

Table 1. Indicators used to analyze participants' behaviors.

\subsection{Interactions with devices}

To analyze low-level interaction, we used log data to count touch events, how many notes participants submitted during the activity, and how many times they pressed the favorite button. We used video analysis to measure the number of touch events, e.g., tap, drag, or zoom, on UI elements of the shared display, in order to measure how active each participant was.

\subsection{Awareness}

To analyze group awareness, we used indicators from Hornecker's et al. model [14]. We took two positive indicators: reaction without explicit request and comple- 
mentary action, which correspond to anticipation and assistance actions. Reaction without request is a proactive action that occurs when one participant reacts to, or helps, another without being explicitly asked. For instance, when a participant sends information about a location to his/her tablet and notices that another group member wants to check the same location, sending the information to him/her without being asked to is considered reaction without request. Complementary action occurred when participants were coordinating the task or distributing labor implicitly. We coded it when two or three participants were interacting together or alternately on the shared display without verbal coordination to achieve the same goal. For example, when two participants were sorting location cards together based on their itinerary, or when they were alternately dragging and zooming the map. These two indicators can be used to evaluate whether participants are aware of the on-going tasks taking place in the group.

We used two negative indicators of awareness: interference and verbal monitoring. Interference occurs when participants unintendedly interrupt or impede another person's actions. For example, when one person wants to choose a location while another accidentally drags the map, or when two participants are reaching for the same location card. Verbal monitoring occurs when a participant is inquiring about other persons' behaviors. For example, when one person is asking the other: "Which location are you checking?"

Finally, we used verbal shadowing to measure and assess how participants' maintained awareness [14]. We coded it when one person was describing or giving a running commentary about who is doing or going to do what. For example, when one person is saying: "I'm writing down the price of that hotel" or "I'll like that location".

\subsection{Activity organization}

We analyzed activity organization in terms of group strategies, explicitly sharing labor, and roles taken by participants $[23,35]$, but also in terms of planning and monitoring [10]. We counted discussions related to activity organization when participants expressed strategies, such as deciding how to distribute labor, e.g. discussing the locations to explore. We also counted this indicator when participants were monitoring or planning these strategies. For example, when a group realized that nobody had favorited the locations explored, one participant stated: "one of us should 'like' the locations so we can filter the cards and find them easily", and another answered: "yeah, you're right, I'll do that". To observe how groups shared labor, we counted parallel interactions, which occurred when participants were interacting together on the large surface for a different purpose.

We were interested in measuring whether the task was more efficient in one condition rather than the other. Quality of results could not be a good efficiency indicator since we proposed an open problem. The different results proposed by the groups all met the budget requirements. Thus, to measure the efficiency of group works, we analyzed the activity in terms of duration and exploration. We used our logs to compute the number of locations explored together and by each person. 


\subsection{Discussion and information sharing}

We coded discussions in video based on the conversation topic. Each time participants talked about a topic within a continuous interval of time, we counted it as one discussion. If two topics were discussed during the same conversation, we counted one discussion per topic. The list of topics was set after the inter-rater reliability test of the two coders' observations. This list included attractions, when participants discussed about places to visit, hotels for discussions about the location of a hotel or room type, budget, when participants were discussing about ticket prices, and itinerary for their plan of the days.

To analyze how participants used their tablets during discussions and how information was shared among the group, we captured each time that they shared their tablets with others. Using a qualitative approach, we observed participants' movements and deictic gestures when exchanging ideas, or arguments when discussing collective decisions.

\subsection{Video analysis process}

Two coders analyzed the videos. We conducted an inter-rater reliability test before starting the analysis. We chose one group from each condition to carry out the test and picked two segments in each group: one at the beginning of the activity when participants were browsing locations, and the other at the end when participants started to discuss their final plan. Each segment lasted 2 minutes. We went through the video twice. In the first round, we noted all the interactions, such as dragging the map, touching cards, tapping the filter buttons, and the complementary or parallel interactions. In the second round, we conducted the verbal analysis, considering the awareness indicators described above, the different types of discussion, etc. In the end, the analysis had $96.46 \%$ agreements (Cohen's Kappa $\kappa=0.88$ ). After we had clarified coding differences and refined our coding scheme, we analyzed all the videos.

\section{$5 \quad$ Results}

We present our results for each of our hypotheses and outline the main findings.

\subsection{Interactions with devices}

We analyzed collaboration among groups from a low-level perspective to determine whether the orientation of the shared display impacted users' interaction. We emitted the hypotheses that horizontal shared display would allow more balanced interaction (H1), whereas combining tablets with the shared display would reduce the differences between conditions in terms of content created or modified (H2).

\section{H1: More equality in physical interaction in the horizontal condition.}

To test this hypothesis, we calculated the percentage of touch events (tap, drag, etc.) per person within each group. We used the same formula as Marshall et al. [20] 
and Potvin et al. [22]. The inequality index is smaller in the horizontal condition (mean $=0.39, \mathrm{SD}=0.15)$ than in the vertical one $($ mean $=0.70, \mathrm{SD}=0.25)$ with a statistically significant difference $(\mathrm{t}(10)=-2.59, \mathrm{p}=0.027)$ (Fig. 3. -left). In the horizontal condition, participants have more balanced interaction. On the other hand, in the vertical condition, groups were always organized around a main participant who had far more interactions than the others (Fig. 3. -right), even though everyone had access to the surface. When the main interactor was interacting, others pointed at the surface to give suggestions or asked the interactor to interact.

We observed behavioral differences on interacting with the large surface. Participants played with the display in the horizontal condition, such as zooming or dragging the map without a clear aim (something Zagermann et al. also noted in their study [35]). In contrast, in the vertical condition, interactions were goal-driven, with participants always touching the display for a specific purpose.
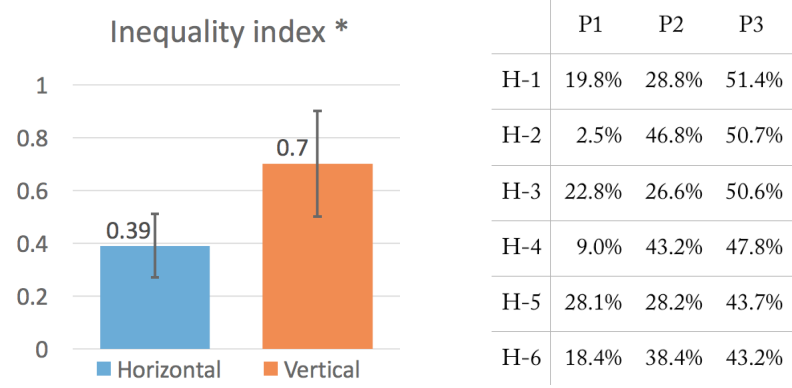

\begin{tabular}{c|ccc} 
& P1 & P2 & P3 \\
\hline V-1 & $8.1 \%$ & $12.2 \%$ & $79.7 \%$ \\
\hline V-2 & $2.0 \%$ & $19.1 \%$ & $78.9 \%$ \\
\hline V-3 & $9.4 \%$ & $14.6 \%$ & $\mathbf{7 6 . 0} \%$ \\
\hline V-4 & $13.8 \%$ & $17.7 \%$ & $\mathbf{6 8 . 5} \%$ \\
\hline V-5 & $19.4 \%$ & $21.3 \%$ & $\mathbf{5 9 . 2} \%$ \\
\hline V-6 & $24.4 \%$ & $28.1 \%$ & $\mathbf{4 7 . 5 \%}$ \\
\hline
\end{tabular}

Fig. 3. More inequality of physical interaction in the vertical condition. Left: inequality index of physical interaction. Right: percentage of touch events per person in two conditions.

Finding 1: these observations validate H1. Large horizontal surfaces support more equality in physical interaction, whereas vertical surfaces lead to the emergence of a main interactor.

\section{H2: Reduction of differences when creating and modifying content.}

To test this hypothesis, we measured the number of notes and favorite locations that each group submitted. The results showed no significant difference between two conditions in notes submitted (horizontal: mean $=16.3, \mathrm{SD}=6.62$, vertical: mean $=$ 11.3, $\mathrm{SD}=8.66 . \mathrm{t}(10)=1.12, \mathrm{p}=0.29)$, or in "Favorites" (horizontal: mean $=22.7$, $\mathrm{SD}=9.4$, vertical: mean $=13.2, \mathrm{SD}=8.7 .(\mathrm{t}(10)=1.81, \mathrm{p}=0.10)$. Unlike previous studies, we did not observe an effect of display orientation on content creation or interaction. Nevertheless, to validate statically this hypothesis, other experiments would need to be conducted with a 'no tablet' condition to compare against.

Finding 2: these observations are in favor of $\mathrm{H} 2$, although more work would be required to validate this hypothesis. Combining tablets with a shared surface could reduce the differences between horizontal and vertical conditions, especially in the creation of and interaction with content, a fact which was highlighted in previous studies [23]. 


\subsection{Group coordination}

To measure whether the orientation of the shared display impacts the way participants collaborate together from a meta-level, we analyzed group coordination according to awareness (H3), activity organization and exploration efficiency (H4) and communication and discussions (H5).

\section{H3: Higher level of awareness in the horizontal condition.}

To test this hypothesis, we looked at several awareness indicators. Verbal monitoring is considered as a negative indicator of awareness [14] and occurs when participants want to know the current situation of the on-going activity, such as what a collaborator is doing or what stage the group is in. We observed few instances of verbal monitoring either in the horizontal condition (mean $=3, \mathrm{SD}=1.67$ ) or the vertical condition $($ mean $=2.83, \mathrm{SD}=1.72)$ (Fig. 4-left). We observed significantly more verbal shadowing in the vertical condition (mean $=11.5, \mathrm{SD}=5.05)$ than in the horizontal condition (mean $=6, \mathrm{SD}=3.1 ; \mathrm{t}(10)=-2.27, \mathrm{p}=0.046)$ (Fig. 4-right). In the vertical condition participants often gave cues to others, such as "I'm going to like that location", "I'll write down the price for that hotel", or "I'm going to look at this attraction to see if it's free".

In both conditions, groups maintained a good level of awareness, and participants did not feel the need to ask what the others were doing. However, in the vertical condition, participants had to make more efforts to maintain this high level of awareness, by explaining to the others what they were doing. This relates to finding 1 and the presence of a main interactor, participants gave more verbal cues to each other to maintain awareness.

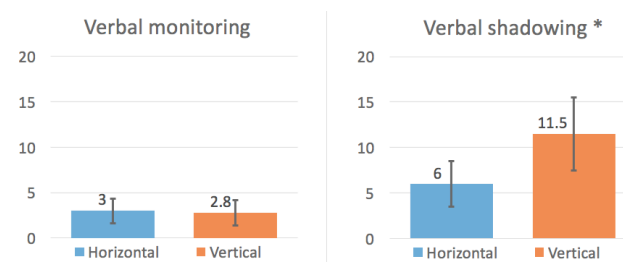

Fig. 4. Average number of verbal monitoring and verbal shadowing statements per condition (means with $95 \% \mathrm{CI}$ ).

Regarding positive indicators of awareness, we observed more reaction without request in the horizontal condition (mean $=8, \mathrm{SD}=3.0$ ) than in the vertical condition (mean $=3.5, \mathrm{SD}=2.1)$. The difference was statistically significant $(\mathrm{t}(10)=-3.01, \mathrm{p}=$ 0.013) (Fig. 5-left). Participants maintained a better awareness of others and offered help without being explicitly asked. For example, in a horizontal condition group, one participant said: "OK, now we can check attractions". Another person then used the filter buttons on the menu bar to hide hotels and show attractions. There were also far more complementary actions in the horizontal condition (mean $=30.8, \mathrm{SD}=10.2$ ) than in the vertical condition (mean $=9.2, \mathrm{SD}=3.9$ ) with a statistically significant difference $(\mathrm{t}(10)=4.85, \mathrm{p}=0.0008)$ (Fig. 5-middle). These actions could be, for 
example, handing over location cards or two participants dragging and zooming the map in turn. Complementary actions mostly occurred when participants were discussing the itinerary and sorting location cards according to their trip plan. More complementary actions suggest that participants were aware of the activity of other people anticipating actions and favoring higher implicit low-level coordination between people [12]. This finding is also related to the fact that there were more balanced interactions in the horizontal condition (Finding 1). As participants interacted equally, there were more chances of their having complementary actions.

We observed a side effect of these balanced interactions in the horizontal condition, there was more interferences in the horizontal condition (mean $=5.5, \mathrm{SD}=3.39)$ than in the vertical condition (mean $=2, \mathrm{SD}=1.67$ ). The difference is statistically significant $(\mathrm{t}(10)=2.27, \mathrm{p}=0.048)$ (Fig. 5-right). As the horizontal condition fosters more balanced interaction, participants were all engaged in interacting, which can in turn cause more interferences: for example, when two participants wanted to drag the map or were reaching for the same location card. In the vertical condition, there was always one main interactor, a fact which prevented interference.

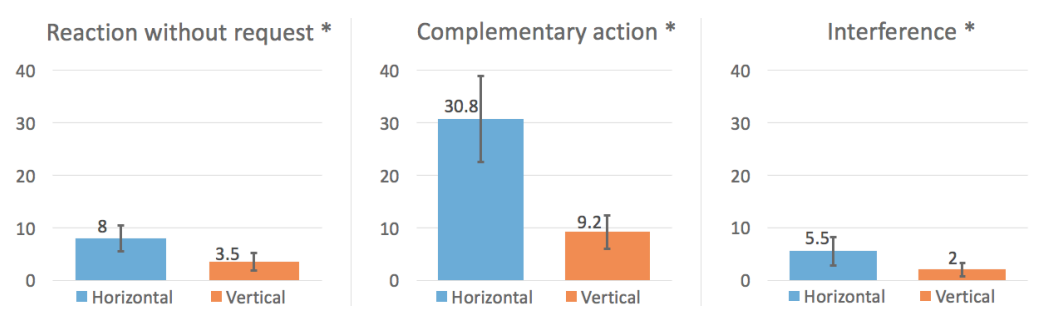

Fig. 5. Average number of reactions without request, complementary actions, and interferences per condition (means with $95 \% \mathrm{CI}$ ).

Finding 3: Groups maintained a good level of awareness in both conditions. In the vertical condition, even if participants made more efforts to maintain awareness, there was little occurrence of implicit coordination. This can be accounted for the emergence of one main interactor handling the large surface (Finding 1). With horizontal displays, participants are more likely to spontaneously help other group members or to finish the actions of others without verbal or explicit synchronization. Consequently, this can lead to more interference since participants interact more with the horizontal surface. Thus, our $\mathrm{H} 3$ hypothesis is partially validated. The horizontal condition offers a sufficiently good level of awareness for implicit coordination such as anticipation and assistance actions.

\section{H4: More efficient activity organization in the horizontal condition.}

To test this hypothesis, we observed how participants organized themselves and discussed good practices, exploration strategies or division of labor.

In both conditions, groups used explicit coordination to reach decisions about strategies or to organize work. We did not observe an impact of surface orientation on the 
number of discussions about strategies between the two conditions (horizontal: mean $=11, \mathrm{SD}=3.52$; vertical: mean $=7.67, \mathrm{SD}=4.08 ; \mathrm{t}(10)=1.51, \mathrm{p}=0.16)$. However, we observed significantly more parallel actions in the horizontal condition (mean = $11, \mathrm{SD}=6.8)$ than in the vertical condition $($ mean $=4.2, \mathrm{SD}=2.99)(\mathrm{t}(10)=2.24, \mathrm{p}=$ 0.048) (Fig. 6-first). For instance, in the horizontal condition, we observed several times a participant organizing the location cards while another person was checking the map. This can be related to our previous findings about balanced interaction (Finding 1) and awareness (Finding 3). Interestingly, in the vertical condition, the effort of maintaining a good level of awareness combined with the emergence of one main interactor for the shared surface did not do away with the need for explicit coordination among participants for strategy and activity organization. Moreover, the main interactor always took control of the activity, thus reducing the potential for parallel actions. In contrast, in the horizontal condition, participants interacted equally and needed to agree explicitly on the activity organization and their strategies. In this condition, more parallel actions were performed on the shared surface.

Regarding exploration strategies and task efficiency, in the horizontal condition, participants preferred to check the same location together (5 out of 6 groups), while in the vertical condition, they distributed labor (5 out of 6 groups). Only one group in each condition did it in the opposite way. Excluding these two opposite groups, the number of location explored simultaneously in the horizontal condition is significantly higher than in the vertical condition. (respectively mean $=20.6, \mathrm{SD}=6.84$, and mean $=7.6, \mathrm{SD}=3.29),(\mathrm{t}(10)=4.20, \mathrm{p}=0.005)($ Fig. 6-second $)$. The simultaneous exploration of locations in the horizontal condition led to significantly $(\mathrm{t}(34)=2.53$, $\mathrm{p}=0.016)$ more locations explored per person in the horizontal condition (mean $=$ 21.6, $\mathrm{SD}=6.84)$ than in the vertical condition (mean $=15.6, \mathrm{SD}=7.4)($ Fig. 6-third).

Finally, even though groups had different exploration strategies in the two conditions, we did not observe a significant difference regarding the time spent on the activity (in horizontal: mean $=28 \mathrm{~m} 06 \mathrm{~s}, \mathrm{SD}=3 \mathrm{~m}$; in vertical: mean $=25 \mathrm{~m} 24 \mathrm{~s}, \mathrm{SD}=$ $5 \mathrm{~m} 48 \mathrm{~s} ; \mathrm{t}(10)=0.98 \mathrm{p}=0.35)$ (Fig. 6-fourth). This suggests that, in the horizontal condition, exploration of location was not as efficient as we expected. Even if participants checked more locations in this condition, they did not reach an agreement on their trip any quicker than in the vertical condition.

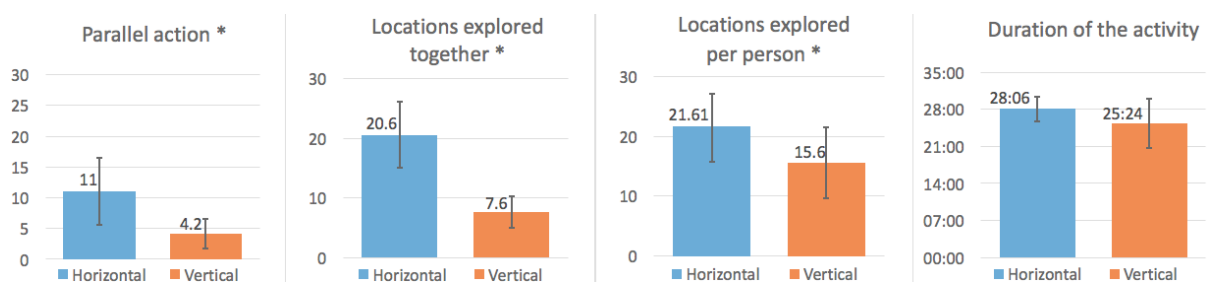

Fig. 6. Average number of parallel actions, locations explored together (excluding two opposite groups), locations explored per person, and duration of the activity (means with 95\% CI). 
Finding 4: Our observations do not validate H4. Participants tended to use two different strategies in the two conditions. In the horizontal condition, groups explored the locations together and checked more locations. While in the vertical condition, participants distributed labor and each person explored fewer locations. In the end, the task duration was similar in both conditions. One strategy was not necessarily more efficient than the other.

H5: More discussions in the horizontal condition.

To test this hypothesis, we analyzed how groups conducted discussions, how people shared information, the devices they used to support the discussion, etc. We analyzed both verbal cues and participants' formations.

The number of discussions that participants had about hotels, attractions, budget and itinerary were similar in both conditions (horizontal: mean $=48.8, \mathrm{SD}=11.3$, vertical: mean $=45.7, \mathrm{SD}=13.5$ ). We initially thought that the design of the activity might have led to this similar number of discussions, and that this number was linked to the number of locations to check. However, participants explored different numbers of locations and adopted different strategies for exploration. Overall, orientation does not seem to influence group discussions.

When discussing a location, participants always used a device - either the shared display or a tablet - to support the discussion and bring in new information. When using the shared display, participants stood close to the screen and pointed at elements such as a marker on the map or a location card. In the vertical condition, participants faced the shared display in a line formation, while in the horizontal condition, they kept the same position throughout the activity. In five out of six groups, two participants stood on the long side and one stood on the short side. In the last group, all participants stood on one long side of the display.

Participants leveraged tablets to introduce new elements into the discussion. In the horizontal condition, probably due to their standing positions around the table, we mostly observed tablet sharing between two participants, such as one person holding his/her tablet towards another, or one person looking over the shoulder of the tablet's owner. We only observed once three participants sharing the same tablet. In the vertical condition, we observed more often three participants sharing the same tablet. Nevertheless, we found no significant difference in the number of times participants shared a tablet (horizontal: mean $=7.17, \mathrm{SD}=3.31$, vertical: mean $=4.5, \mathrm{SD}=4.23 ; \mathrm{t}$ $(10)=1.22, \mathrm{p}=0.25)$.

When the discussion did not rely on the shared surface, such as when participants calculated the cost of their trip, participants in the vertical condition always changed their position to a circular arrangement to maintain face-to-face discussion. Either the participant in the middle would step back, or the two participants on the side would

Finding 5: We did not find a significant difference between the two conditions in the number of discussions within groups. This invalidates our hypothesis of discussions being better supported in the horizontal condition. In both conditions, participants used tablets for sharing information or individually exploring information, to bring arguments into the discussion. Furthermore, unlike in previous work [1], we observed participants re-arranging their formation, and forming more triad formations in the vertical condition. 
step forward. In the horizontal condition, only the group with three participants standing in line changed its position, with the person in the middle stepping back a slightly. Other groups kept their former positions during the discussion.

\section{Discussion}

We studied the impact of display orientation in MSE by replicating and extending previous studies on single displays, in a realistic context, with modern high resolution multi-touch displays. Previous work on single displays is in places contradictory, our results weigh in, confirm or contradict them in places, and extend them (see Table 2). Building on our results and contrasting them to previous, we now look at how display orientation shaped collaboration. We use these results to draw implications for the design of collaborative activities in MSE.

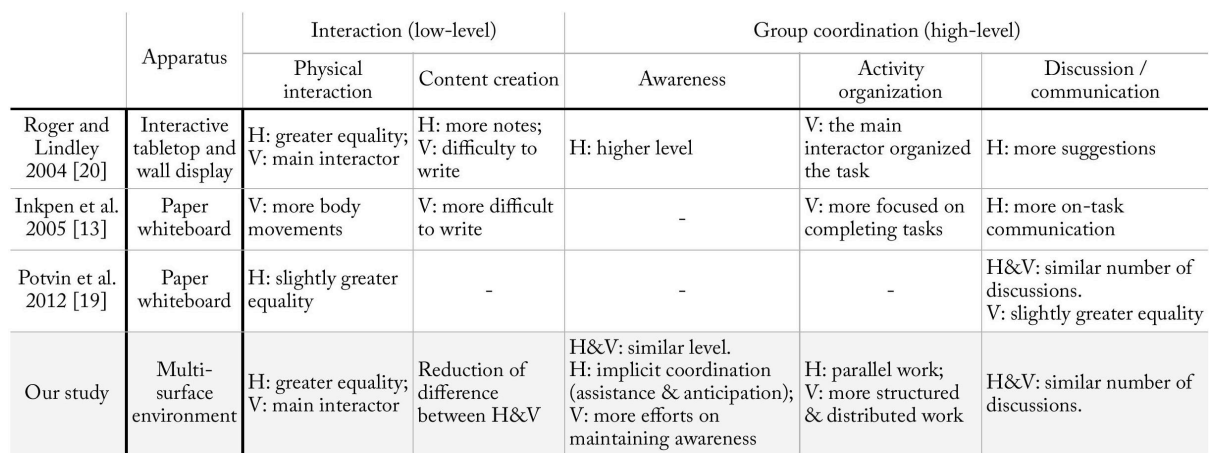

Table 2. Comparison of findings from former studies with ours. $\mathrm{H}$ and $\mathrm{V}$ respectively stand for horizontal and vertical conditions.

\subsection{Creating and interacting with content}

Our study confirms previous results on the impact of display orientation on interaction $[22,23]$. In the horizontal condition, physical interaction with the surface was more equally distributed among group members. The use of tablets does not seem to significantly impact direct interaction with the large surface. However, when looking at interactions that involved inputting information, tablets proved to be particularly beneficial in the vertical condition. Content creation trends toward similarity in the two conditions. On a qualitative level, participants valued more the introduction of tablets in the vertical condition than in the horizontal condition. This suggests that MSE reduces differences previously observed in single shared display set-ups.

\subsection{Similar level of awareness and discussions.}

Awareness mechanisms and their consequences on the activity were ignored by Inkpen et al. and Potvin et al. [15], and only briefly analyzed by Rogers and Lindley [23]. 
In our study, we analyzed awareness in detail, distinguishing positive and negative indicators. Unlike in previous work [23], we found that participants maintained a good level of awareness in both conditions, as the low number of verbal monitoring suggests. However, in the vertical condition, this awareness came at a cost, as participants used far more verbal shadowing, i.e., announcing what they do. Moreover, they kept moving backward to observe the situation, before moving forward to analyze information on the shared display. In our study, the MSE set-up does not seem to impact communication with a number of discussions similar in both conditions (which contradict Rogers and Lindley [23] but confirms Potvin et al. [22]).

\subsection{Surface orientation influences the activity organization.}

Compared to previous work, we observed more signs of implicit and explicit coordination in the horizontal condition. Participants were more inclined to anticipate the actions of others and to share and agree on strategies or good practices to conduct the task. The activity seemed to run far more naturally, as participants took upon themselves to visit locations and to support each other. However, participants had to make more efforts to ensure that they were in sync. In contrast, distribution of work seemed more efficient in the vertical condition. The person who interacted most with the display also distributed labor and sent information to others (also observed in Rogers and Lindley [23]). Even if participants took collective decisions all the same, the activity seemed more structured, with less interference than in the horizontal condition.

\subsection{MSE set-ups shape F-formations}

Our MSE set-up shaped how participants positioned themselves. In the horizontal condition, participants mostly maintained their formation, merely tilting their tablets to show their content to another participant. On the other hand, in the vertical condition, tablet sharing led to changes in position (often semi-circular or side-by-side formation). This suggests that bringing tablets could introduce freedom in group activities. Participants would have a personal workspace to conduct individual exploration, and join group discussions when needed.

\subsection{Implications for design}

Our analysis indicated that the surface orientation induced participants to organize their activities in different ways. Horizontal surfaces seem to support more cohesive collaborative activities where participants go through the task together. On the other hand, vertical surfaces seem better suited to cooperative situations in which one person drives an activity and distributes tasks to others. Therefore, we suggest choosing a horizontal surface for activities that require equal participation, such as collaborative learning where participants have to acquire the same skills and knowledge [2]. We suggest using a vertical surface for the activities where one person takes control. Software features could also be considered to facilitate the task organization and dis- 
tribution, such as providing opportunities for the main interactor to change the contents on others personal devices.

Incorporating indicators on the devices (e.g. showing who is exploring which location) could help improve awareness and decrease the amount of monitoring required. These visual feedbacks could also encourage participants to regulate their activity and mitigate the influence of screen orientation. Besides, if activities involve individual content creation, such as adding comments and doing individual exploration, then introducing handheld devices in complement to the shared surface should lead to more balanced contributions among participants.

In the end, building on the F-formations observed, we suggest to use proxemic interactions for the activities to support discussion, especially the activities involved with rich content or data. Cross-device interaction should support micro-mobile behaviors and support exchange of complex information across devices, such as transfer content or duplicate screens [20].

\subsection{Limitations}

The set-up used for our study is just one instance within the much wider space of MSE, and further studies are needed to build a solid body of knowledge on the topic. To reduce the number of confounding factors, all participants stood up. This could be questioned since tabletops might be used more widely in a seated position. Participants started the activity working side by side, and while UI elements could be rotated, they all had the same orientation to begin with.

\section{Conclusion}

MSE are particularly suited to supporting collaborative activities, enabling dynamic combination of devices to support group activities on large shared surfaces and individual activities on personal devices. Although there is a wealth of application examples demonstrating the benefits of MSE, the underlying collaborative dynamics are still unclear. We conducted a study showing that the orientation of a shared display in a MSE shapes group coordination. Our results show that MSE reduces differences between the horizontal and vertical conditions when it comes to create and interact with content. More importantly, it profoundly shapes the way collaborative activities are conducted: using a horizontal surface will lead to better equity of interaction and more cohesive activities. On the other hand, group coordination is more structured and is organized around a main interactor when a vertical display is used.

\section{Acknowledgement}

We thank all our participants. This work was partially funded by the China Scholarship Council PhD program and the ANR project JENlab (ANR-13-APPR-0001). 


\section{$9 \quad$ References}

1. AlTarawneh, R., Jaber, R.N., Humayoun, S.R. and Ebert, A., 2015, November. Collaborative Position Patterns for Pairs Working with Shared Tiled-Wall Display using Mobile Devices. In Proceedings of the 2015 International Conference on Interactive Tabletops \& Surfaces (ITS'15) (pp. 259-264). ACM.

2. Al-Megren, S., Kharrufa, A., Hook, J., Holden, A., Sutton, S., \& Olivier, P. 2015. Comparing Fatigue when Using Large Horizontal and Vertical Multi-Touch Interaction Displays. In Human-Computer Interaction (pp. 156-164). Springer International Publishing.

3. Bachour, K., Kaplan, F. and Dillenbourg, P., 2010. An interactive table for supporting participation balance in face-to-face collaborative learning. IEEE Transactions on Learning Technologies, 3(3), pp.203-213.

4. Bell, G. and Dourish, P., 2007. Yesterday's tomorrows: notes on ubiquitous computing's dominant vision. Personal and ubiquitous computing, 11(2), pp.133-143.

5. Campos, P., Ferreira, A., \& Lucero, A. (2013, October). Collaboration meets interactive surfaces: walls, tables, tablets, and phones. In Proceedings of the 2013 ACM international conference on Interactive tabletops and surfaces (ITS'13) (pp. 481-482). ACM.

6. Chokshi, A., Seyed, T., Marinho Rodrigues, F. and Maurer, F., 2014, November. ePlan multi-surface: A multi-surface environment for emergency response planning exercises. In Proceedings of the Ninth ACM International Conference on Interactive Tabletops and Surfaces (ITS'15) (pp. 219-228). ACM.

7. Clark, H.H. and Brennan, S.E., 1991. Grounding in communication. Perspectives on socially shared cognition, 13(1991), pp.127-149.

8. DiMicco, J.M., Pandolfo, A. and Bender, W., 2004, November. Influencing group participation with a shared display. In Proceedings of the 2004 ACM conference on Computer supported cooperative work (CSCW'04) (pp. 614-623). ACM.

9. Döring, T., Shirazi, A.S. and Schmidt, A., 2010, May. Exploring gesture-based interaction techniques in multi-display environments with mobile phones and a multi-touch table. In AVI (Vol. 10, pp. 419-419).

10. Evans, A.C., Wobbrock, J.O. and Davis, K., 2016, February. Modeling Collaboration Patterns on an Interactive Tabletop in a Classroom Setting. In Proceedings of the 19th ACM Conference on Computer-Supported Cooperative Work \& Social Computing (pp. 860871). ACM.

11. Fleck, R., Rogers, Y., Yuill, N., Marshall, P., Carr, A., Rick, J. and Bonnett, V., 2009, November. Actions speak loudly with words: unpacking collaboration around the table. In Proceedings of the ACM international conference on interactive tabletops and surfaces (ITS'15) (pp. 189-196). ACM.

12. Gutwin, C. and Greenberg, S., 2002. A descriptive framework of workspace awareness for real-time groupware. Computer Supported Cooperative Work (CSCW), 11(3-4), pp.411446.

13. Hall, E.T., 1966. The hidden dimension. Doubleday, Garden City, NY

14. Hornecker, E., Marshall, P., Dalton, N.S. and Rogers, Y., 2008, November. Collaboration and interference: awareness with mice or touch input. In Proceedings of the 2008 ACM conference on Computer supported cooperative work (CSCW'08) (pp. 167-176). ACM.

15. Inkpen, K., Hawkey, K., Kellar, M., Mandryk, R., Parker, K., Reilly, D., Scott, S. and Whalen, T., 2005, July. Exploring display factors that influence co-located collaboration: angle, size, number, and user arrangement. In Proc. HCI international (Vol. 2005). 
16. Luff, P. and Heath, C. 1998. Mobility in collaboration. In Proceedings of the 1998 ACM conference on Computer supported cooperative work (CSCW '98). ACM, New York, NY, USA, 305-314.

17. Kendon, A., 1990. Conducting interaction: Patterns of behavior in focused encounters (Vol. 7). CUP Archive.

18. Malone, T.W. and Crowston, K., 1990, September. What is coordination theory and how can it help design cooperative work systems?. In Proceedings of the 1990 ACM conference on Computer-supported cooperative work\} (CSCW'90) (pp. 357-370). ACM.

19. Marquardt, N., Hinckley, K. and Greenberg, S., 2012, October. Cross-device interaction via micro-mobility and f-formations. In Proceedings of the 25th annual ACM symposium on User interface software and technology (pp. 13-22). ACM.

20. Marshall, P., Hornecker, E., Morris, R., Dalton, N.S. and Rogers, Y., 2008, October. When the fingers do the talking: A study of group participation with varying constraints to a tabletop interface. In Horizontal Interactive Human Computer Systems, 2008. TABLETOP 2008. 3rd IEEE International Workshop on (pp. 33-40). IEEE.

21. Perry, M., O'hara, K., Sellen, A., Brown, B. and Harper, R., 2001. Dealing with mobility: understanding access anytime, anywhere. ACM Transactions on Computer-Human Interaction (TOCHI), 8(4), pp.323-347.

22. Potvin, B., Swindells, C., Tory, M. and Storey, M.A., 2012. Comparing horizontal and vertical surfaces for a collaborative design task. Advances in Human-Computer Interaction, 2012, p.6.

23. Rogers, Y. and Lindley, S., 2004. Collaborating around vertical and horizontal large interactive displays: which way is best?. Interacting with Computers, 16(6), pp.1133-1152.

24. Schmidt, K., 2002. The problem with 'awareness': Introductory remarks on 'awareness in CSCW'. Computer Supported Cooperative Work (CSCW'02), 11(3-4), pp.285-298.

25. Seyed, T., Costa Sousa, M., Maurer, F., and Tang, A. (2013). Skyhunter: a multi-surface environment for supporting oil and gas exploration. In Proceedings of the 2013 ACM international conference on Interactive tabletops and surfaces (ITS'13), pages 15-22. ACM.

26. Scott, S.D., Besacier, G., Tournet, J., Goyal, N. and Haller, M., 2014, November. Surface ghosts: promoting awareness of transferred objects during pick-and-drop transfer in multisurface environments. In Proceedings of the Ninth ACM International Conference on Interactive Tabletops and Surfaces (ITS'14) (pp. 99-108). ACM.

27. Scott, S.D., Grant, K.D. and Mandryk, R.L., 2003. System guidelines for co-located, collaborative work on a tabletop display. In ECSCW 2003 (pp. 159-178). Springer Netherlands.

28. Sugimoto, M., Hosoi, K. and Hashizume, H., 2004, April. Caretta: a system for supporting face-to-face collaboration by integrating personal and shared spaces. In Proceedings of the SIGCHI conference on Human factors in computing systems (CHI'04) (pp. 41-48). ACM.

29. Vasiliou, C., Ioannou, A., \& Zaphiris, P. 2015, September. An artifact ecology in a nutshell: A distributed cognition perspective for collaboration and coordination. In HumanComputer Interaction (pp. 55-72). Springer International Publishing.

30. Vauras, M., Iiskala, T., Kajamies, A., Kinnunen, R. and Lehtinen, E., 2003. Sharedregulation and motivation of collaborating peers: A case analysis. Psychologia, 46(1), pp.19-37.

31. Volet, S., Vauras, M. and Salonen, P., 2009. Self-and social regulation in learning contexts: An integrative perspective. Educational psychologist, 44(4), pp.215-226.

32. Wallace, J.R., Scott, S.D., Stutz, T., Enns, T. and Inkpen, K., 2009. Investigating teamwork and taskwork in single-and multi-display groupware systems. Personal and Ubiquitous Computing, 13(8), pp.569-581. 
33. Weiser, M., 1991. The computer for the 21 st century. Scientific american, 265(3), pp.94104.

34. Yuill, N. and Rogers, Y., 2012. Mechanisms for collaboration: A design and evaluation framework for multi-user interfaces. ACM Transactions on Computer-Human Interaction (TOCHI), 19(1), p.1, 25 pages.

35. Zagermann, J., Pfeil, U., Rädle, R., Jetter, H.C., Klokmose, C., Reiterer, H., 2015. When Tablets meet Tabletops: The Effect of Tabletop Size on Around-the-Table Collaboration with Personal Tablets. In Proceedings of the 34th annual ACM conference on Human factors in computing systems (CHI'16) (Vol. 13, pp. 53-67). ACM. 